\title{
Schematic concept formation: Demonstration in a free sorting task
}

\author{
SELBY H. EVANS AND MALCOLM D. ARNOULT \\ TEXAS CHRISTIAN UNIVERSITY
}

Presented with a set of patterns containing two schemadefined categories, Ss were asked to sort the patterns into two categories. No instructions about relevant attributes were given, and no feedback was provided. A significant number of Ss used the schema-defined categories in sorting.

Schematic concept formation (SCF) has been proposed as a construct of schema theory (Evans, 1967a). SCF was there defined as the development of the ability to assign objects to their corresponding schema families on the basis of information derived from perceiving the objects, without any other source of information and without prior familization with the relevant schemata. (In this context, a schema is a rule describing a prototype, and a schema family is a population of objects which may be efficiently described in terms of deviations from the prototype.)

Clearly SCF requires demonstration before it can claim status as a construct of schema theory. An experiment by Shipstone (1960) showed that in a free sorting task, in which Ss were asked to sort instances from several finite-state grammars, the Ss tended to use the generating grammars as the basis of sorting. But these grammars were deterministic; schema theory requires sorting competence with instances which incorporate deviations from the schema. Edmonds, Mueller, \& Evans (1966) used such instances and showed that, without KR, schema-defined categories exerted an increasing influence on judgments of similarity and difference. Although this result supports the SCF hypothesis, the experiment does not explicitly show category formation. We here report research designed specifically to show that Ss could and would use schema-defined categories in a free sorting task, i.e., a task which required them to sort patterns into categories without instruction about relevant attributes and without knowledge of results. Stimuli

The basic requirement for demonstrating SCF is a capability for introducing arbitrarily defined and unfamiliar schemata into populations of patterns in such a way that the schema can be manipulated (that is, one schema can be substituted for another) without altering other potentially relevant variables. The VARGUS 7 pattern generating system (Evans, 1967b) was designed to meet that objective. The system produces each pattern by generating a segment of a specialized Markov process and mapping its elements into column heights to construct a histoform pattern. The schema inheres in a set of transitional probabilities distributed so that each column height has a most probable successor. The set of most probable successors defines a most probable sequence (MPS), or schema. The MPS can be altered without altering such potentially relevant variables as the average area of the patterns, the average number of columns of any particular height, and the probability of any particular column height at any particular location.

Two MPSs were selected by random permutations of the elements $1,2,3,5,6$, and 7 . Selection of the second MPS was so constrained that none of its transitions duplicated those of the first. For all favored transitions, the probability was .75. Transitions to or from Element 4 had a probability of $1 / 7$ (in order to introduce further randomness into the patterns), while all other transitions had a probability of .021. For each MPS, 30 patterns, 30 elements in length, were generated and printed on an IBM 407 with columns represented by $\mathrm{Xs}$. The resulting 60 patterns were ordered in a constrained random permutation such that each block of 10 patterns had five patterns from either MPS.

To insure that results would not be specific to a particular pair of MPSs or order of presentation, four pairs of MPSs were formed by the above procedure and for each pair of MPSs, seven different orders of presentation were used.

\section{Subjects}

Ss were 105 undergraduates at Texas Christian University, enrolled in various psychology and mathematics courses. The task was presented to an entire class at a time, each student being given a booklet and an answer sheet.

Instructions

The following instructions were read:

This is an investigation to find out how well people can do at distinguishing different kinds of patterns. In each booklet there are 60 patterns and each pattern is different, but each booklet has just two different kinds of patterns. We will call them Type $A$ and Type B. What I am going to ask you to do is go through your booklet and classify each pattern according to whether you think it an $A$ or B. Every booklet has about the same number of As as of Bs. Naturally, when you start you will simply be guessing, but as you look at more and more of the patterns you will begin to have a better idea of which pattern is an A and which is a $B$. No single detail of a pattern will serve as a clue and no simple rule will work. You will probably do your best if you work rather quickly and rely on your first impression. If you are not sure, make a guess and go on. 
Further instructions stipulated that patterns had to be classified in the order in which they were encountered and reassured Ss that the task was not a test of their ability.

Scoring

No single response could be scored as correct or incorrect because Ss were free to assign the responses $A$ and $B$ to either schema family. This freedom required scoring each S separately; SCF would be demonstrated by consistency in assigning a given letter to members of the same schema family. The last 20 responses of each $S$ were scored by summing the number of times Schema 1 was called " $A$ " and the number of times Schema 2 was called "B." A sum near 20 would indicate a tendency to use the schemadefined categories and so would a sum near zero (this result would imply that $S$ was consistently calling Schema 1 " $B$ " and Schema 2 "A"). If S were categorizing independently of the schema-defined categories, the sum would be near 10. It has been shown (Evans, 1964) that the null hypothesis of independent sorting can be rejected with a satisfactory small probability of error (.041) if the sum is five or less, or if the sum is 15 or greater. This rule was used to decide, for each $S$, whether he was using the schema-defined categories in his classifications.

\section{Resulis and Conclusions}

Of 105 Ss, 22 were found to have met the criterion of consistent categorizing. This number is small enough to raise the question of whether it could have occurred by chance. The answer is easily determined by reference to the binomial distribution with $\mathrm{N}=105$ and $\mathrm{p}=$ .041. Reference to the Poisson approximation to this distribution (Molina, 1949) shows that less than .005 of the distribution lies above 10. Thus the number of consistent Ss far exceeds the number that could be expected by chance. Further support for the conclusion that these 22 Ss were using the schema-defined categories was obtained by scoring the first 40 responses of each of these Ss in terms of consistency with the categorizing rule which the $\mathrm{S}$ was apparently using on the last 20 trials. Reference to the binomial distribution in this case allowed rejection $(p<.0001)$ of the hypothesis that these Ss were sorting independently of the schema-defined categories.

Consistent sorting was not specific to MPS or to order of presentation. All four pairs of MPSs yielded some Ss who were consistent, and 15 of the 28 orders of presentation were represented among the consistent Ss. That the majority of Ss did not satisfy the criterion of the decision rule is in part attributable to a rather high probability of Type 2 error resulting from the relatively small number (20) of observations on which the decision was based. On the other hand, it is likely that a substantial number of Ss did not use the schema-defined categories.

Two replications of this experiment, with certain modifications, have corroborated the above results. In these replications, the patterns were reprinted by an improved printing process (see Evans, 1967b). The task was described as a test of the ability to learn to recognize patterns. The notion of unaided sorting was illustrated in the instructions by the following: Suppose someone gave you a collection of handwriting samples of two different people. Even if the samples were all mixed together without any means to identify them, you could look over the samples for a while and then probably sort them into categories according to the person who wrote them.

In the first replication, Ss were tested in large groups, and $25 \%$ were found to be consistent (as contrasted with $21 \%$ in the original study). In the second replication, 27 Ss were tested ${ }^{2}$ in very small groups (about three Ss) and $44 \%$ were found to be consistent, an improvement which appears to have been the result of increased interest.

We conclude that at least some Ss can and will use schema-defined categories in a free sorting task. This conclusion derives further support from a recently completed study by Rosser (1967), who used a free sorting task with sequences of tones as stimuli. In this study also, a Markov process was used to introduce a pattern into the stimuli, and Ss were found to use this pattern when categorizing stimuli from a set consisting of patterned and random sequences.

Thus SCF is demonstrable phenomenon. It has, in fact, been demonstrated in several other pieces of research (Brown, Walker, \& Evans, 1967; Tracy \& Evans, 1967). The inclusion of SCF as a component of schema theory thus appears to be justified.

\section{References}

BROWN, B. R., WALKER, D. W., \& EVANS, S. H. Discrimination among schemata as a function of amount of pretraining and constraint redundancy. Paper read at Southwest. Psychol. Assoc., Houston, April, 1967.

EDMONDS, E. M., MUELLER, M. R., \& EVANS, S. H. Effects of knowledge of results on mixed schema discrimination. Psychon. Sci. 1966 $6,377-378$

EVANS, S. H. A model for perceptual category formation. Unpublished doctoral dissertation, Texas Christian University, 1964.

EVANS, S. H. A brief statement of schema theory. Psychon. Sci, 1967a, 8, 87-88.

EVANS, S. H. VARGUS 7: Computed patterns from Markov processes. Behav. Sci. 1967b, 12, 323-328.

MOLINA, E. C. Poisson's exponential binomial limit. New York: Van Nostrand, 1949.

ROSSER, E. M. Categorization and discrimination of tone sequences. Unpublished doctoral dissertation, Harvard, 1967.

SHIPSTONE, E. I. Some variables affecting pattern conception. Psychol. Monogr., 1960, 74, No. 17 (Whole No. 504).

TRACY, J.K., \& EVANS, S. H. The effects of supplementary information on schema learning. Paper read at Southwest. Psychol. Assoc., Houston, April, 1967.

Notes

1. This research is a part of the first author's doctoral dissertation, submitted at Texas Christian University in 1964 and in part supported by a NSF Cooperative Graduate Fellowship. Additional support was provided by the TCU Computer Center and TCU Research Foundation Grant No. PS 6672. A number of details are omitted from the necessarily brief report.

2. I thank Mr. William C. Scott for assistance at this point. 